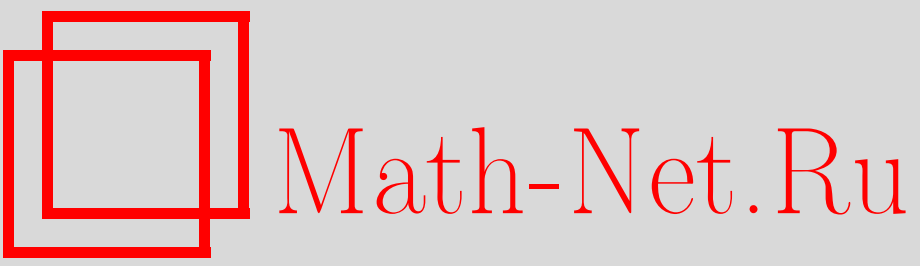

М. Бальдо, В. С. Ольховский, Аналитические свойства $S$-матрицы для взаимодействий с "хвостами" в виде потенциала Юкавы, ТМФ, 2006, том 147, номер 1, 113-128

DOI: https://doi.org/10.4213/tmf2027

Использование Общероссийского математического портала Math-Net.Ru подразумевает, что вы прочитали и согласны с пользовательским соглашением

http: //www . mathnet.ru/rus/agreement

Параметры загрузки:

IP : 54.196 .121 .252

26 апреля 2023 г., 17:45:07 
ТЕОРЕТИЧЕСКАЯ

И МАТЕМАТИЧЕСКАЯ

ФИЗИКА

Том 147, № 1

апрель, 2006

(C) 2006 г.

М. Бальдо*, В. С. Ольховский ${ }^{\dagger}$

\section{АНАЛИТИЧЕСКИЕ СВОЙСТВА $S$-МАТРИЦЫ ДЛЯ ВЗАИМОДЕЙСТВИЙ С "ХВОСТАМИ" В ВИДЕ ПОТЕНЦИАЛА ЮКАВЫ}

Получено явное аналитическое выражение для $S$-матрицы при произвольном центральном взаимодействии внутри сферы конечного радиуса для "хвоста" в виде потенциала Юкавы на больших расстояниях. Метод использует полноту волновых функций вне конечной сферы, а также условия унитарности и симметрии $S$-матрицы.

Ключевые слова: $S$-матрица, центрально-симметричное взаимодействие, потенциал Юкавы.

\section{1. ВВЕДЕНИЕ}

В настоящее время аналитическая структура нерелятивистской одноканальной $S$-матрицы достаточно хорошо известна для широкого класса сферических взаимодействий (см., например, книги [1], [2] и работы [3]-[5]). По-видимому, только случай взаимодействий с "хвостами" в виде потенциала Юкавы, для которого явные аналитические представления $S$-матриц неизвестны, представляет собой существенное исключение. Действительно, этот случай характерен для ядерной физики - как для нуклон-нуклонных, так и для нуклон-ядерных взаимодействий. Цель настоящей работы состоит в том, чтобы на основании метода, развитого в работах [3]-[5], до некоторой степени восполнить этот пробел.

\section{2. ОСНОВНЫЕ ФОРМУЛЫ И ОБЩИЕ СВОЙСТВА $S$-МАТРИЦЫ}

Предположим, что взаимодействие между двумя сталкивающимися частицами вне сферы радиуса $a(a<\infty)$ описывается центральным потенциалом Юкавы

$$
V=V_{0}\left[(b r)^{-1} e^{-b r}\right], \quad V_{0}<0, \quad b^{-1} \sim a,
$$

*I.N.F.N., Sezione di Catania, Catania, Italy. E-mail: baldo@ct.infn.it

${ }^{\dagger}$ Институт ядерных исследований НАН Украины, Киев, Украина. E-mail: olkhovsk@kinr.kiev.ua 
в то время как внутри сферы оно имеет произвольный вид.

Радиальную волновую функцию рассеяния $R_{l}^{(+)}$при граничных условиях, содержащих расходящуюся волну, для $l$-й парциальной волны и волнового числа $k$ при $r>a$ можно записать в виде

$$
R_{l}^{(+)}(k, r)=\frac{i}{2 k r}\left[f_{l-}(k, r) e^{i l \pi / 2}-S_{l}(k) f_{l+}(k, r) e^{-i l \pi / 2}\right]
$$

Радиальную волновую функцию $R_{l}^{n}$ для связанного состояния в той же области можно записать в виде

$$
R_{l}^{n}\left(k_{n l}, r\right)=(2 \pi)^{-1 / 2} \frac{B_{l}\left(k_{n l}\right) f_{l+}\left(k_{n l}, r\right)}{r},
$$

где $k_{n l}$ - соответствующее собственное значение, $B_{l}$ - так называемая постоянная связанного состояния. $S$-матрица, или, более точно, функция $S_{l}(k)$ в уравнении $(2)$, удовлетворяет (расширенному) условию унитарности

$$
S_{l}(k) S_{l}^{*}\left(k^{*}\right)=1
$$

и условию симметрии

$$
S_{l}(k) S_{l}(-k)=1
$$

(см., например, [1]-[5]). Определения и некоторые свойства функций $f_{l \pm}(k, r)$ представлены в монографии [1]. Вся информация о взаимодействии при $r<a$ содержится в функциях $S_{l}(k)$, постоянных $B_{l}\left(k_{n l}\right)$ и числах $k_{n l}$.

Рассмотрим аналитические свойства функций $f_{l \pm}(k, r)$ для потенциалов Юкавы (1). Прежде всего, согласно [1] для случая $l=0$ имеем

$$
f_{0 \pm}(k, r)=\left[1+\int_{b}^{\infty} S_{ \pm}\left(b^{\prime}, k\right) e^{-b^{\prime} r} d b^{\prime}\right] e^{ \pm i k r},
$$

где $S_{ \pm}\left(b^{\prime}, k\right)$ - решение уравнения

$$
b(b \mp 2 i k) S_{ \pm}(b, k)=\rho+\int_{b}^{\infty} \rho S_{ \pm}\left(b^{\prime}, k\right) d b^{\prime}
$$

где $\rho=2 \mu V_{0} /\left(\hbar^{2} b^{3}\right)<0, \quad \mu$ - приведенная масса. Решение уравнения (7) можно записать в виде

$$
S_{ \pm}(b, k)=\rho\left\{b(b \mp 2 i k)\left[1 \mp \frac{i \rho}{2 k} \ln \left(1 \mp \frac{2 i k}{b}\right)\right]^{-1}\right\},
$$

тогда из уравнения (6) получаем

$$
f_{0 \pm}(k, r)=\left\{1+\rho\left[1 \mp \frac{i \rho}{2 k} \ln \left(1 \mp \frac{2 i k}{b}\right)\right]^{-1} \int_{b}^{\infty} \frac{e^{-b^{\prime} r} d b^{\prime}}{b^{\prime}\left(b^{\prime} \mp 2 i k\right)}\right\} e^{ \pm i k r}
$$


В уравнении (8) имеется логарифмическая особенность функции $f_{0 \pm}(k, r)$ в точке $k=k_{\gamma}=i b / 2$. Рассмотрим аналитические свойства множителя

$$
A_{-}=\left[1+\frac{i \rho}{2 k} \ln \left(1+\frac{2 i k}{b}\right)\right]^{-1} .
$$

Легко проверить, что при комплексном значении $k$, если как $\operatorname{Re} k$, так и $\operatorname{Im} k$ отличны от нуля, множитель $A_{-}^{-1}$ не имеет нулей, и поэтому $A_{-}$не имеет полюсов. Тот же результат получается при вещественных $k$. Положим далее $k=i x$, где $x-$ вещественное число, и перепишем выражение (9) в виде

$$
A_{-}=\left[1+\frac{\rho}{2 x} \ln \left(1-\frac{2 x}{b}\right)\right]^{-1} .
$$

В случае $2 x / b>1$ множитель $A_{-}^{-1}$ не имеет нулей, поскольку логарифм является комплексным, и поэтому $A_{-}$не имеет полюсов. В случае $0 \leqslant 2 x / b<1$ при $\rho<0$, как и для дальнодействующей части ядерных сил, множитель $A_{-}$снова не имеет полюсов. Наконец, в случае $2 x / b \leqslant 0$ полюсы могут существовать, однако они должны быть расположены в нижней полуплоскости комплексной плоскости $k$.

В заключение заметим, что множитель $A_{-}$не содержит дополнительных сингулярностей в верхней полуплоскости комплексной плоскости $k$, за исключением точки ветвления при $k_{\gamma}=i b / 2$.

Рассуждения можно распространить на более высокие значения момента импульса $l>0$. Та же логарифмическая особенность в точке $k=k_{\gamma}=i b / 2$ появляется также и для функции $f_{l-}(k, r)$. Чтобы это показать, можно воспользоваться интегральным уравнением, которое позволяет вычислить функцию $f_{l-}(k, r)$ исходя из функции $f_{0-}(k, r)$ :

$$
f_{l-}(k, r)=f_{0-}(k, r)+l(l+1) \int_{r}^{\infty} G\left(k ; r, r^{\prime}\right)\left(r^{\prime}\right)^{-2} f_{l-}\left(k, r^{\prime}\right) d r^{\prime},
$$

где $r>a$ и функция Грина $G\left(k ; r, r^{\prime}\right)$ имеет вид [1]

$$
\begin{aligned}
G\left(k ; r, r^{\prime}\right) & =(2 i k)^{-1}\left[f_{0-}(k, r) f_{0+}\left(k, r^{\prime}\right)-f_{0-}\left(k, r^{\prime}\right) f_{0+}(k, r)\right]= \\
& =\left[f_{0+}(k, 0)\right]^{-1}\left[\Phi(k, r) f\left(k, r^{\prime}\right)-\Phi\left(k, r^{\prime}\right) f(k, r)\right] .
\end{aligned}
$$

Поскольку функция $\Phi(k, r)$ всюду регулярна, функция Грина в уравнении (11) не имеет особенности в точке $k$. Решение $f_{l-}(k, r)$ уравнения (10) при любом значении $l$ содержит ту же логарифмическую особенность в точке $k_{\gamma}=i b / 2$, что и функция $f_{0-}(k, r)$.

После этих предварительных замечаний рассмотрим аналитическую структуру $S$-матрицы $S_{l}(k)$. Для этого используем, как и в работах [3], [5], условие полноты волновых функций в области $r \geqslant a$. Мы предполагаем, что в этой внешней области сталкивающиеся частицы могут описываться с помощью уравнения Шредингера 
только с одним потенциалом Юкавы (возможно, с добавлением потенциала, убывающего быстрее, чем любая экспонента). Поэтому при $r, r^{\prime}>a$ можно записать

$$
\frac{2}{\pi} \int_{0}^{\infty} k^{2} R_{l}^{(+)}(k, r) R_{l}^{(+) *}\left(k, r^{\prime}\right) d k+\sum_{n} R_{l}^{(n)}\left(k_{n l}, r\right) R_{l}^{(n) *}\left(k_{n l}, r^{\prime}\right)=r^{-2} \delta\left(r-r^{\prime}\right) .
$$

Используя хорошо известное свойство [1]

$$
f_{l+}^{*}(k, r)=f_{l-}(k, r), \quad f_{l-}^{*}(k, r)=f_{l+}(k, r),
$$

которое выполняется для вещественных $k$, и условия (4) и (5), уравнение (12) можно записать в виде

$$
\begin{gathered}
\frac{1}{2 r^{\prime}} \int_{C} f_{l-}(k, r) f_{l+}\left(k, r^{\prime}\right) d k-\frac{(-1)^{l}}{r r^{\prime}} \int_{C} S_{l}(k) f_{l+}(k, r) f_{l+}\left(k, r^{\prime}\right) d k+ \\
+\frac{1}{r r^{\prime}} \sum_{n}\left(B_{l}\left(k_{n l}\right)\right)^{2} f_{l+}\left(k_{n l}, r\right) f_{l+}\left(k_{n l}, r^{\prime}\right)=\frac{2 \pi \delta\left(r-r^{\prime}\right)}{r^{2}} .
\end{gathered}
$$

Путь интегрирования $C$ в уравнении (14) проходит вдоль вещественной оси от $-\infty$ до $\infty$, кроме окрестности начала координат $k=0$, где он проходит по полуокружности бесконечно малого радиуса в верхней полуплоскости $D^{+}$. В точке $k=0$ функции $f_{l \pm}(k, r)$ имеют полюсы порядка $l$.

Поскольку для выбранного потенциала во внешней области $(r>a)$ функции $f_{l \pm}(k, r)$ имеют пределы $e^{ \pm i k r}$ при $|k| \rightarrow \infty$ по всем направлениям в комплексной плоскости, можно преобразовать путь интегрирования внутри $D^{+}$к другому пути, состоящему из двух частей: произвольно большой полуокружности, расположенной выше вещественной оси, и набора контуров внутри $D^{+}$, обходящих вокруг всех существующих особенностей, разрезов или полюсов сколь угодно близко к ним. После такого преобразования, используя тождества

$$
\begin{gathered}
\int_{\Gamma^{+}} f_{l-}(k, r) f_{l+}\left(k, r^{\prime}\right) d k=\int_{\Gamma^{+}} e^{i k\left(r^{\prime}-r\right)} d k=\int_{-\infty}^{\infty} e^{i k\left(r^{\prime}-r\right)} d k=2 \pi \delta\left(r-r^{\prime}\right), \\
\int_{\Gamma^{+}} S_{l}(k) f_{l+}(k, r) f_{l+}\left(k, r^{\prime}\right) d k=\int_{\Gamma^{+}} S_{l}(k) e^{i k\left(r^{\prime}+r\right)} d k
\end{gathered}
$$

получим [5]

$$
\begin{aligned}
\sum_{m} \oint_{k_{m}} f_{l-}(k, r) f_{l+}\left(k, r^{\prime}\right) d k+\sum_{p} \oint_{\gamma_{p}} f_{l-}(k, r) f_{l+}\left(k, r^{\prime}\right) d k- \\
-(-1)^{l} \sum_{s} \oint_{k_{s}} S_{l}(k) f_{l+}(k, r) f_{l+}\left(k, r^{\prime}\right) d k- \\
-(-1)^{l} \sum_{q} \oint_{\gamma_{q}} S_{l}(k) f_{l+}(k, r) f_{l+}\left(k, r^{\prime}\right) d k- \\
-(-1)^{l} \int_{\Gamma^{+}} S_{l}(k) e^{i k\left(r^{\prime}+r\right)} d k+\sum_{n}\left(B_{l}\left(k_{n l}\right)\right)^{2} f_{l+}\left(k_{n l}, r\right) f_{l+}\left(k_{n l}, r^{\prime}\right)=0 .
\end{aligned}
$$


Здесь $\Gamma^{+}$- бесконечно большая полуокружность, расположенная выше вещественной оси, $k_{m}, k_{s}$ обозначают изолированные особенности, а $\gamma_{p}, \gamma_{q}$ - контуры, которые окружают неизолированные особенности или края разреза в случае точек ветвления. Поскольку функции $f_{l+}(k, r)$ и $e^{i k r}$ независимы как функции $r$ в различных точках $k_{n l}, k_{m}, k_{s}, \gamma_{p}, \gamma_{q}$ (являющихся особыми точками для функций $f_{l-}(k, r)$ и $\left.S_{l}(k)\right)$ в $D^{+}$, уравнение $(16)$ эквивалентно следующей системе уравнений:

$$
\begin{aligned}
-(-1)^{l} \oint_{k_{n l}} S_{l}(k) f_{l+}(k, r) f_{l+}\left(k, r^{\prime}\right) d k & =\left(B_{l}\left(k_{n l}\right)\right)^{2} f_{l+}\left(k_{n l}, r\right) f_{l+}\left(k_{n l}, r^{\prime}\right), \\
(-1)^{l} \oint_{k_{m}} S_{l}(k) f_{l+}(k, r) f_{l+}\left(k, r^{\prime}\right) d k & =\oint_{k_{m}} f_{l-}(k, r) f_{l+}\left(k, r^{\prime}\right) d k, \\
(-1)^{l} \oint_{\gamma_{p}} S_{l}(k) f_{l+}(k, r) f_{l+}\left(k, r^{\prime}\right) d k & =\oint_{\gamma_{p}} f_{l-}(k, r) f_{l+}\left(k, r^{\prime}\right) d k, \\
\int_{\Gamma^{+}} S_{l}(k) e^{i k\left(r^{\prime}+r\right)} d k & =0, \quad r, r^{\prime} \geqslant a .
\end{aligned}
$$

Согласно теореме о вычетах из уравнения (17) можно заключить, что $S_{l}(k)$ может иметь в $D^{+}$полюсы первого порядка, соответствующие связанным состояниям, с вычетами, равными $(-1)^{l+1} i(2 \pi)^{-1}\left(B_{n l}\right)^{2}$. Уравнение $(18)$ можно переписать в виде

$$
\oint_{k_{m}} f_{l+}(k, r) f_{l+}\left(k, r^{\prime}\right)\left[\frac{f_{l-}\left(k, r^{\prime}\right)}{f_{l+}\left(k, r^{\prime}\right)}-(-1)^{l} S_{l}(k)\right] d k=0
$$

откуда можно заключить, что $S_{l}(k)$ может иметь в $D^{+}$дополнительные изолированные особенности, которые совпадают с изолированными особенностями функции $f_{l-}(k, r)$ в $D^{+}$, вблизи которых

$$
\lim _{k \rightarrow k_{m}} f_{l-}(k, r)=\lim _{k \rightarrow k_{m}} D_{m}(k) f_{l+}(k, r) .
$$

Функция $D_{m}(k)$ не зависит от $r$ и имеет изолированную особенность в точке $k_{m}$. Эти результаты были получены в [1] с использованием другого метода. В приложении 1 представлен перевод на русский язык работы Ольховского и Цехмистренко [6], где представлены подобные результаты относительно полюсов для более общих, чем в [1], взаимодействий, опубликованной ранее в украинском издании, которая не была переведена на русский и английский языки и поэтому неизвестна читателям, не знающим украинского языка.

Теперь рассмотрим уравнение (19) в окрестности логарифмических особенностей функции $f_{l-}(k, r)$ в $D^{+}$. Контур $\gamma_{q}$ можно выбрать в виде, показанном на рис. 1 . Он состоит из почти замкнутой окружности $\gamma_{\text {асс }}$, проведенной вокруг точки $k_{\gamma}=i b / 2, \mathrm{c}$ малым радиусом $\varepsilon \equiv(\varepsilon / b) b$ и двух бесконечных линий $\gamma_{\mathrm{sh}}$ вдоль краев разреза с намного меньшим расстоянием между ними, равным $(\varepsilon / b)^{\delta} b, \delta>2$, т.е. $\gamma_{q}=\gamma_{\mathrm{acc}}+\gamma_{\mathrm{sh}}$. Обозначим через $\gamma_{12}$ сегмент, соединяющий низшие для двух линий точки 1 и 2

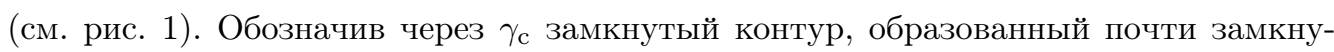
той окружностью и сегментом, для интегралов, входящих в уравнение (19), можно 


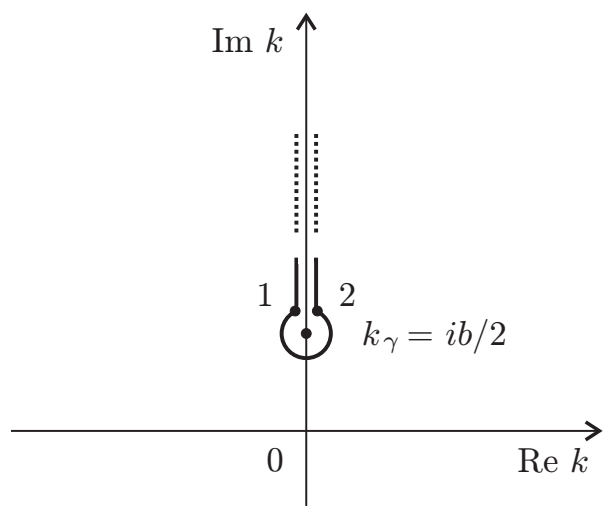

Рис. 1. Вид контура $\gamma_{q}$.

записать тождество

$$
\int_{\gamma_{\mathrm{acc}}}=\oint_{\gamma_{\mathrm{c}}}-\int_{\gamma_{12}} \underset{\varepsilon \rightarrow 0}{\longrightarrow} \oint_{\gamma_{\mathrm{c}}}
$$

Поскольку длина сегмента $\gamma_{12}$ равна $(\varepsilon / b)^{\delta} b$, интегралы по $\gamma_{12}$, так же как и по $\gamma_{\mathrm{sh}}$, при $\varepsilon \rightarrow 0$ стремятся к нулю как $O\left(\varepsilon^{\delta-2}\right)$. Поэтому остается только контурный интеграл вдоль замкнутой окружности $\gamma_{\mathrm{c}}$ с центром в точке $k_{\gamma}$.

Рассмотрим подробно интеграл по $\gamma_{c}$. Значение интеграла определяется поведением подынтегрального выражения, когда радиус окружности стремится к нулю. Поэтому рассмотрим предел

$$
\lim _{k \rightarrow k_{\gamma}}\left[1+\frac{i \rho}{2 k} \ln \left(1+\frac{2 i k}{b}\right)\right]^{-1} \int_{b}^{\infty} \frac{e^{\left(i k-b^{\prime}\right) r}}{b^{\prime}\left(b^{\prime}+2 i k\right)} d b^{\prime} .
$$

Легко показать, что интеграл по переменной $b^{\prime}$ в этом выражении имеет в точке $k_{\gamma}$ логарифмическую расходимость, которая сокращается при обращении в нуль соответствующего множителя, и поэтому функция $f_{0-}(k, r)$ не имеет полюса в точке $k_{\gamma}$. Явное вычисление предела (23) показывает, что в окрестности точки $k_{\gamma}$ функцию $f_{0-}(k, r)$ можно записать в виде (см. приложение 2)

$$
f_{0-}(k, r) \rightarrow W(k, r)+\left[1+\frac{i \rho}{2 k} \ln \left(1+\frac{2 i k}{b}\right)\right]^{-1} U(k, r)
$$

где $W$ и $U$ - аналитические функции переменной $k$ в точке $k_{\gamma}$ и внутри малой замкнутой окружности $\gamma_{c}$. Поэтому уравнение (19) можно переписать в виде

$$
\begin{aligned}
& \oint_{\gamma_{\mathrm{c}}} S_{0}(k) f_{0+}(k, r) f_{0+}\left(k, r^{\prime}\right) d k=\oint_{\gamma_{\mathrm{c}}} f_{0+}(k, r) f_{0-}\left(k, r^{\prime}\right) d k= \\
& =\oint_{\gamma_{\mathrm{c}}} W(k, r) f_{0+}\left(k, r^{\prime}\right) d k+\left[1+\frac{i \rho}{2 k} \ln \left(1+\frac{2 i k}{b}\right)\right]^{-1} \oint_{\gamma_{\mathrm{c}}} U(k, r) f_{0+}\left(k, r^{\prime}\right) d k
\end{aligned}
$$


Поскольку каждый интеграл в правой части обращается в нуль, можно заключить, чTо

$$
\oint_{\gamma_{\mathrm{c}}} S_{0}(k) f_{0+}(k, r) f_{0+}\left(k, r^{\prime}\right) d k=0
$$

Отсюда следует, что $S_{0}(k)$ может содержать не более, чем сингулярный множитель вида

$$
F=\left[1-\frac{i \rho}{2 k} \ln \left(1-\frac{2 i k}{b}\right)\right]\left[1+\frac{i \rho}{2 k} \ln \left(1+\frac{2 i k}{b}\right)\right]^{-1},
$$

связанный с аналогичной логарифмической точкой ветвления при $k=k_{\gamma}$. Конечно, такого множителя может и не быть или $S_{0}(k)$ может содержать другие множители, имеющие логарифмическую точку ветвления, но обращающиеся в нуль в точке $k_{\gamma}$.

Рассмотрим несколько специальных случаев, когда этот множитель действительно появляется.

Если взаимодействие внутри сферы $r \leqslant a$ таково, что волновую функцию рассеяния во внешней части $(r>a)$ можно записать в виде

$$
\Psi_{\text {ext }}=f_{0-}(k r)-S_{0}(k) f_{0+}(k r)
$$

причем она обращается в нуль в некоторой точке $r=r_{0}>a$, то

$$
S_{0}(k)=\frac{f_{0-}\left(k, r_{0}\right)}{f_{0+}\left(k, r_{0}\right)} .
$$

Отсюда следует, что в $S_{0}(k)$ должен содержатъся множитель $F$, задаваемый выражением (27).

Другая возможность возникает для широкого класса потенциалов [1], а именно когда взаимодействие внутри сферы $r \leqslant a$ таково, что выполняются следующие соотношения непрерывности:

$$
\begin{aligned}
\Psi_{\mathrm{int}} & \equiv \text { const } \cdot \Phi(k, a)=f_{0-}(k, a)-S_{0}(k) f_{0+}(k, a), \\
\left.\frac{d \Psi_{\mathrm{int}}}{d r}\right|_{r=a} & \left.\equiv \mathrm{const} \cdot \frac{d \Phi(k, r)}{d r}\right|_{r=a}=\left.\frac{d f_{0-}(k r)}{d r}\right|_{r=a}-\left.S_{0} \frac{d f_{0+}(k, r)}{d r}\right|_{r=a} .
\end{aligned}
$$

Здесь функция $\Phi(k, r)$ представляет собой регулярное решение радиального уравнения Шредингера внутри сферы $r \leqslant a$ с граничными условиями $\Phi(k, 0)=0$. Эта функция определяется только взаимодействием внутри сферы $r \leqslant a$. Уравнения (30) определяют константу и соответствующую $S$-матрицу:

$$
S_{0}(k)=\frac{\varphi(k, a) d f_{0-}(k, a) / d a-f_{0-}(k, a) d \varphi(k, a) / d a}{f_{0+}(k, a) d \varphi(k, a) / d a-\varphi(k, a) d f_{0+}(k, a) / d a},
$$

т.е. тогда в $S_{0}(k)$ также должен содержаться множитель $(27)$.

Согласно соотношениям $(10),(11)$ тот же результат получается и для $S_{l}(k)$ при $l>0$. 


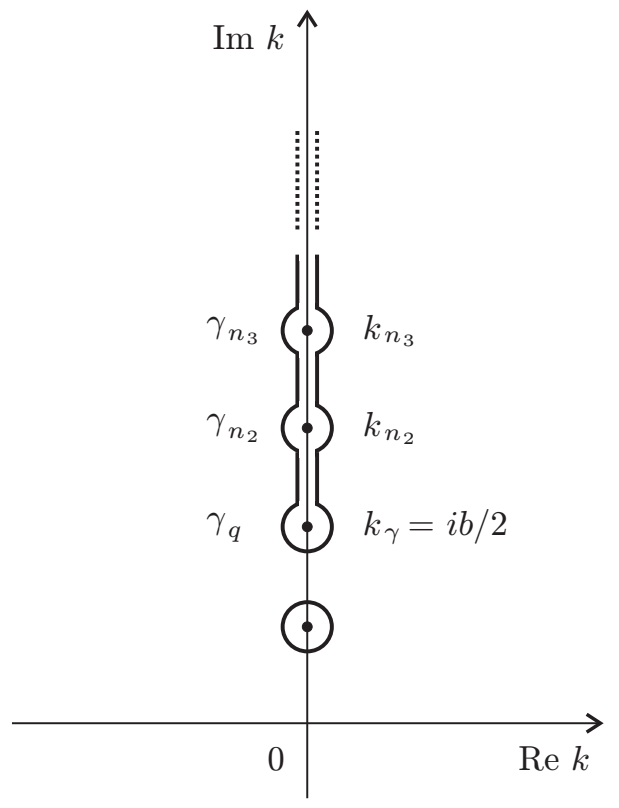

Рис. 2. Наложение полюсов и разреза.

С помощью такого же подхода можно исследовать более общий случай, когда имеется накрытие (см. рис. 2) разреза полюсами (теми, которые соответствуют связанным состояниям и/или "лишним" полюсам, которые появляются, когда потенциалы экспоненциально убывают). В данном случае достаточно использовать аналогичные соотношению (22) равенства

$$
\int_{\gamma_{\mathrm{acc}}\left(k_{n}\right)}=\oint_{k_{n}}-\int_{\gamma_{12}} \underset{\varepsilon \rightarrow 0}{\rightarrow} \oint_{k_{n}}
$$

и только повторить рассуждения, приведенные перед формулой (24). Тогда легко доказать, что для всех этих особенностей равенство (21) остается справедливым, и поэтому остаются справедливыми полученные ранее результаты относительно особенностей $S_{l}(k)$ в $D^{+}$.

Наконец, можно найти аналитическое продолжение функций $S_{l}(k)$ в нижнюю полуплоскость $D^{-}$, как это обычно делается, на основании условия симметрии (5) и известной общей теоремы об аналитическом продолжении.

\section{3. ЯВНОЕ АНАЛИТИЧЕСКОЕ ПРЕДСТАВЛЕНИЕ $S$-МАТРИЦЫ ДЛЯ ВЫБРАННОГО КЛАССА ВЗАИМОДЕЙСТВИЙ}

Теперь попробуем найти явное аналитическое представление функции $S_{l}(k)$. Множитель $F$, определяемый в (27), содержит логарифмические особенности, аналогичные особенностям решения (8), в точках $k_{b}=i b / 2$ и $-k_{b}$, удовлетворяет условию 
симметрии (5), имеет на вещественной оси $k$ абсолютное значение, равное единице, и, наконец, не имеет других особенностей, кроме, быть может, нуля в знаменателе в (27), и не имеет нулей, кроме, быть может, нуля в числителе в (27).

Учитывая вид уравнения (25) и равенство (20), легко видеть, что функции $\widetilde{S}_{l}^{(F)}(k)=F^{-1} \widetilde{S}_{l}(k)$ при $\widetilde{S}_{l}(k)=S_{l}(k) e^{2 i k a}$ не содержат ни логарифмических особенностей, ни нулей, ни полюсов, связанных с наличием логарифмического множителя $(27)$, для любого значения $l$. Они являются регулярными и ограниченными всюду в верхней полуплоскости (включая вещественную ось), кроме точек $k_{n l}{ }^{1)}$. А для функции $\widetilde{S}_{l}^{(F)}(k)$ можно найти разложение в произведение, следуя подходу, который был намечен в общих чертах в работе [5] и получил дальнейшее развитие в настоящей работе.

В первую очередь, мы рассмотрим некоторые промежуточные произведения. Произведение

$$
\prod_{n} \frac{k+k_{n l}}{-k+k_{n l}}
$$

содержит все полюсы функции $S_{l}(k)$ в $D^{+}$, не имеет других особенностей и нулей, удовлетворяет условиям (4) и (5) и имеет абсолютное значение, равное единице на вещественной оси $k$.

Произведение

$$
\prod_{v} \frac{-k+k_{v l}}{k+k_{v l}}
$$

содержит все нули функции $S_{l}(k)$ на положительной части мнимой оси (кроме, конечно, нулей функции $F$ ) и согласно условиям (4) и (5), которые выполнены для выражения (33), содержит также все полюсы функции $S_{l}(k)$ на отрицательной части мнимой оси, имеет абсолютное значение, равное единице на вещественной оси $k$, и в случае сходимости является регулярным в $D^{+}$.

Произведение

$$
\prod_{s} \frac{\left(k_{s l}-k\right)\left(k_{s l}^{*}+k\right)}{\left(k_{s l}+k\right)\left(k_{s l}^{*}-k\right)}
$$

содержит все нули функции $S_{l}(k)$ в $D^{+}$(кроме мнимой оси и нулей функции $F$ ), удовлетворяет условиям (4) и $(5)$ и в случае сходимости является регулярным в $D^{+}$ и имеет абсолютное значение, равное единице на вещественной оси $k$.

Функция

$$
J_{l N}(k)=\widetilde{S}_{l}^{(F)}(k)\left[\prod_{n} \frac{k+k_{n l}}{-k+k_{n l}} \prod_{v}^{N_{1}} \frac{-k+k_{v l}}{k+k_{v l}} \prod_{s}^{N_{2}} \frac{\left(k_{s l}-k\right)\left(k_{s l}^{*}+k\right)}{\left(k_{s l}+k\right)\left(k_{s l}^{*}-k\right)}\right]^{-1}
$$

для конечных чисел $N=N_{1}+N_{2}$ является регулярной и ограниченной на вещественной оси $k$. Если предел

$$
J_{l}(k)=\lim _{N \rightarrow \infty} J_{l N}(k)
$$

\footnotetext{
1) Возможный нуль в числителе $(27)$ исключен из функции $\widetilde{S}_{l}^{(F)}(k)$.
} 
существует, он обладает теми же свойствами, и тогда

$$
S_{l}(k)=e^{-2 i k a} F(k) \prod_{n} \frac{k+k_{n l}}{-k+k_{n l}} \prod_{v} \frac{-k+k_{v l}}{k+k_{v l}} \prod_{s} \frac{\left(k_{s l}-k\right)\left(k_{s l}^{*}+k\right)}{\left(k_{s l}+k\right)\left(k_{s l}^{*}-k\right)} .
$$

Для того чтобы быть уверенными в справедливости (корректности) соотношения (36), требуется показать, что два бесконечных произведения в (36) сходятся. Условием их абсолютной сходимости является сходимость суммы

$$
\begin{aligned}
\sum_{s} & {\left[\left|\frac{k_{s l}-k}{k_{s l}+k}-1\right|+\left|\frac{k_{s l}^{*}+k}{k_{s l}^{*}-k}-1\right|\right]+\sum_{v}\left|\frac{k_{v l}-k}{k_{v l}+k}-1\right|=} \\
& =2|k|\left\{\sum_{s} \frac{1}{\left|k+k_{s l}\right|}+\sum_{s} \frac{1}{\left|k_{s l}^{*}-k\right|}+\sum_{v} \frac{1}{\left|k+k_{v l}\right|}\right\} .
\end{aligned}
$$

В свою очередь, сходимость формулы (37) определяется сходимостью суммы

$$
2 \sum_{s} \frac{1}{\left|k_{s l}\right|}+\sum_{v} \frac{1}{\left|k_{v l}\right|}
$$

поскольку $\left|k_{s l}\right| \rightarrow \infty$ при $s \rightarrow \infty$ и $\left|k_{v l}\right| \rightarrow \infty$ при $v \rightarrow \infty$. Легко убедиться в сходимости сумм (38), если принять во внимание, что функция

$$
\tilde{J}_{l}(k)=\widetilde{S}_{l}^{(F)}(k) / \prod_{n} \frac{k+k_{n l}}{-k+k_{n l}}
$$

является аналитической в $D^{+}$при отсутствии нулей у нее выше вещественной оси $k$, и использовать следующую теорему.

ТЕОРЕма [7]. Пусть функиия $f(z)$ является ограниченной и аналитической при $\operatorname{Re} z \geqslant 0$ и пусть ее нулями в правой полуплоскости z являются $r_{1} e^{\theta_{1}}, r_{2} e^{\theta_{2}}, \ldots$. Тогда ряд $\sum_{n=1}^{\infty} r_{n}^{-1} \cos \theta_{n}$ сходится.

Поскольку для $\tilde{J}_{l}(\rho)$ при $\rho=i k$ справедливо $\cos \theta_{n}=\left|\cos \theta_{n}\right| \geqslant \varepsilon$, где $\varepsilon \neq 0$, то

$$
\varepsilon \sum_{n=1}^{\infty} r_{n}^{-1}<\sum_{n=1}^{\infty} r_{n}^{-1} \cos \theta_{n}<\infty
$$

что доказывает сходимость сумм (38). Таким образом, бесконечные произведения в выражении (36) равномерно сходятся и дают мероморфную функцию с полюсами $-k_{s l}$ и $-k_{v l}$.

Следует заметить, что для всех известных взаимодействий в области ядерной физики и физики элементарных частиц "резонансные" нули $S$-матрицы (в одноканальном пределе) при возрастании $k$ всегда расположены на возрастающих расстояниях от вещественной оси $k$ (и поэтому на возрастающих расстояниях от мнимой оси $\rho$ для $\left.\tilde{J}_{l}(\rho)\right)$. Таким образом, нижняя граница $\varepsilon$ величины $\cos \theta_{n}$ при $n \rightarrow \infty$ оказывается ненулевой и не слишком маленькой, и поэтому сумма $\sum_{n=1}^{\infty} r_{n}^{-1}$ является не только конечной, но также и не очень большой, по меньшей мере пропорциональной $\varepsilon^{-1}$. 
Более того, для всех взаимодействий, для которых справедлива теорема Левинсона, суммы (37) должны сходиться. Это прямое следствие неположительности (отрицательности или нулевого значения) интеграла $\int_{0}^{\infty} \Delta \tau(E) d E, \Delta \tau(E)=(\hbar / 2) \times$ $\left(\partial \arg S_{l} / \partial E\right) E$ - время задержки, $\hbar=h /(2 \pi), h-$ постоянная Планка, на всей положительной полуоси кинетических энергий $E=\hbar^{2} k^{2} /(2 \mu)$ относительного движения сталкивающихся частиц [8], [9], поскольку вклад "резонансных" нулей $k_{s}$ и нулей "виртуальных состояний" $k_{v}$ в интеграл $\int_{0}^{\infty} \Delta \tau(E) d E$ положителен, и при этом он меньше, чем абсолютное значение вклада конечного числа полюсов, соответствующих связанным состояниям, и членов, связанных с отражением (от границ взаимодействия).

Возвращаясь к функции $J_{l}(k)$, заметим, что она не только является аналитической функцией в $D^{+}$, но и, будучи целой функцией без нулей, может быть записана в виде $e^{u+i v}$, где $u+i v$ - целая функция (см., например, соответствующую теорему в работе [7]). Вещественная функция $u(k)$ должна быть отрицательной в $D^{+}$вследствие равенства $(20)$ и положительной в $D^{-}$вследствие условий (4) и (5). Поэтому согласно уравнениям Коши-Римана на вещественной оси $k$ должно выполняться условие

$$
0=\frac{\partial u}{\partial \operatorname{Im} k}=-\frac{\partial v}{\partial k}, \quad \operatorname{Im} k=0 .
$$

Отсюда следует, что функция $v(k)$ монотонно возрастает и принимает любое вещественное значение не более одного раза. Тогда функция $u+i v$ принимает любое мнимое значение не более одного раза и, следовательно, должна быть линейной функцией $k$ :

$$
u+i v=2 i \alpha_{1} k+\alpha_{2} .
$$

Очевидно, $\alpha_{1}=0$, и вследствие равенства $S_{l}(0)=1$ мы имеем $\alpha_{2}=0$. Таким образом, окончательно получаем

$$
S_{l}(k)=e^{-2 i k \alpha} F(k) \prod_{n} \frac{k+k_{n l}}{-k+k_{n l}} \prod_{v} \frac{-k+k_{v l}}{k+k_{v l}} \prod_{s} \frac{\left(k_{s l}-k\right)\left(k_{s l}^{*}+k\right)}{\left(k_{s l}+k\right)\left(k_{s l}^{*}-k\right)},
$$

где $\alpha=a-\alpha_{1} \leqslant a$, а множитель $F$ определяется соотношением $(27)$.

\section{4. ЗАКЛЮЧЕНИЕ}

Формула (42) получена впервые и является прямым обобщением результатов работ [3]-[5] для взаимодействий с “хвостом" в виде потенциала Юкавы. В свою очередь, в дальнейшем, используя методы, представленные в настоящей работе и в работе [5], ее можно легко обобщить на случаи нецентральных нарушающих четность взаимодействий и взаимодействий с поглощением. Исследованию многоканального рассеяния для случая взаимодействий с “хвостом" в виде потенциала Юкавы будет посвящена отдельная работа.

Следует заметить, что во всех видах дисперсионных соотношений требуется учитывать вычеты не только в полюсах $k_{n l}$, но и в “лишних" полюсах для экспоненци- 
ально убывающего потенциала, а также интегралы по контурам $\gamma_{q}$ вокруг логарифмических особенностей.

ПРИЛОЖЕНИЕ 1

Необходимое и достаточное условия существования "лишних" полюсов $(m / 2) i b$ при $b>0, m=1,2, \ldots$ у функции $f_{0}(k, r)$, а поэтому и у $S_{0}(k)^{2)}$

Используя уравнение Йоста

$$
f_{0}(k, r)=e^{i k r}+k^{-1} \int_{r}^{\infty} \sin \left[k\left(r^{\prime}-r\right)\right] V\left(r^{\prime}\right) f_{0}\left(k, r^{\prime}\right) d r^{\prime}
$$

и решая его формально методом последовательных приближений, получим сумму

$$
f_{0}(k, r)=\sum_{v=0}^{\infty} f_{0 v}(k, r)
$$

где

$$
\begin{gathered}
f_{00}(k, r)=e^{i k r} \\
f_{0 v}(k, r)=e^{i k r}(2 i k r)^{v} \int_{r}^{\infty}\left(e^{2 i k\left(r_{1}-r\right)}-1\right) V\left(r_{1}\right) \times \cdots \\
\cdots \times \int_{r_{v-1}}^{\infty} e^{-2 i k\left(r_{v}-r_{v-1}\right)} V\left(r_{v}\right) d r_{v} \ldots d r_{1}, \quad v=1,2, \ldots,
\end{gathered}
$$

для случаев, когда [10] $|V|<M / r^{2+\delta}, M<\infty, \delta>0$. Далее используем следующие теоремы.

Теорема 1 [7]. Пусть $F(k, r)$ - функиия комплексных переменных $k$ u $r$, которая определена и непрерывна для всех значений $k$ в некоторой области $D$ и для всех значений $r$ на контуре $C$. Тогда функция

$$
\Phi(k)=\int_{C} F(k, r) d r
$$

является аналитической функцией $k$ в области D. Если контур $C$ является бесконечным, то необходимой также является равномерная сходимость интеграла.

ТеОрема 2 [7]. Пусть все функции ряда $u_{1}(z), u_{2}(z), \ldots$ - аналитические функции переменной $z$ внутри области $D$, а сумма $\sum_{n=1}^{\infty} u_{n}(z)$ равномерно сходится в каждой области $D^{\prime}$, внутренней по отношению $\kappa$ D. Тогда функиия $u(z)=$ $\sum_{n=1}^{\infty} u_{n}(z)$ представляет собой аналитическую функиию аргумента z внутри D.

Из уравнения (П.3) и теоремы 1 следует, что в верхней полуплоскости функции $f_{0 v}(k, r), v=0,1,2, \ldots$, являются аналитическими функциями переменной $k$. Если борновский ряд (П.2) равномерно сходится, то согласно теореме 2 функция $f_{0}(k, r)$ аналитична в верхней полуплоскости. Аналогично функция $f_{0}(-k, r)$ аналитична в

2) Здесь представлен посвященный памяти Ю. В. Цехмистренко перевод с украинского языка работы [6]. 
нижней полуплоскости. Более того, если функция $f_{0 v}(k, r), v \geqslant 1$, аналитична в нижней полуплоскости, то все последующие члены также являются аналитическими.

Теперь мы покажем, что справедлива следующая теорема.

ТЕорема 3. Если функиия $f_{0 v}(k, r), v>1$, имеет особенности, то функиия $f_{01}(k, r)$ тоже должна их иметь.

Действительно, пусть функция $f_{01}(k, r)$ всюду аналитична. Тогда все последующие члены тоже всюду аналитичны. Это противоречие доказывает теорему 3.

Используем аналитическую структуру функции $f_{01}(k, r)$ в верхней полуплоскости. Очевидно, задача может быть сведена к изучению аналитической структуры интеграла

$$
I=\int_{r}^{\infty} e^{-2 i k r^{\prime}} V\left(r^{\prime}\right) d r^{\prime},
$$

поскольку все остальные члены дают функции, аналитические на всей плоскости.

Как было показано в работах [10], [11], в случае потенциала

$$
V(r)=P_{n}(r) e^{-b r},
$$

где $P_{n}(r)$ - полином $n$-й степени, $b>0$, функция $f_{0}(-k, r)$ имеет полюсы порядка не выше $n+1$ в точках $i b / 2, i b, 3 i b / 2, \ldots$ и является аналитической во всех других точках комплексной плоскости.

Покажем теперь, что если функция $f_{01}(-k, r)$ имеет полюс порядка не выше $n+1$ в точке $i b / 2$, то потенциал должен содержать член вида (П.4). Из этого условия следует, что интеграл

$$
I_{1}=\int_{r}^{\infty} e^{-2 i k r^{\prime}} V_{1}\left(r^{\prime}\right) d r^{\prime}
$$

где $V_{1}=V-V_{2}$ (член $V_{2}$ не дает полюсов), можно представить на действительной оси $k$ в виде

$$
I_{1} \equiv \sum_{\mu=0}^{n} \frac{\varphi_{\mu}(-2 i k, r)}{(2 i k+b)^{\mu}},
$$

где $\varphi_{\mu}(-2 i k, r)$ - функция, аналитическая по всем полюсам и не равная нулю в точке $i b / 2$. Далее, переписывая интеграл $I_{1}$ в виде

$$
I_{1}=\int_{r}^{\infty} e^{-(2 i k+b) r^{\prime}} V_{1}\left(r^{\prime}\right) e^{b r^{\prime}} d r^{\prime}
$$

и интегрируя по частям, мы можем преобразовать правую часть тождества (П.5) в ряды:

$$
\begin{aligned}
-e^{-2 i k r} V_{1} & -\frac{1}{2 i k+b} e^{-2 i k r}\left(b V_{1}+\frac{d V_{1}}{d r}\right)-\cdots-\frac{1}{(2 i k+b)^{n}} e^{-2 i k r} \times \\
\times & \left(b^{n} V_{1}+n b^{n-1} \frac{d V_{1}}{d r}+\cdots+\frac{d^{n} V_{1}}{d r^{n}}\right)-\cdots \equiv \sum_{\mu=0}^{n} \frac{\varphi_{\mu}(-2 i k, r)}{(2 i k+b)^{\mu}} .
\end{aligned}
$$


Сравнивая коэффициенты при равных степенях $(2 i k+b)$, получаем последовательную систему соответствующих тождеств. Поскольку в правой части (П.6) отсутствует член, содержащий $(2 i k+b)^{-m}, m>n$, получаем

$$
b^{n+1} V_{1}+(n+1) b^{n} \frac{d V_{1}}{d r}+\frac{(n+1) n}{2}-b^{n-1} \frac{d^{2} V_{1}}{d r^{2}}+\cdots+\frac{d^{n+1} V_{1}}{d r^{n+1}}=0
$$

откуда следует

$$
V=P_{n}(r) e^{-b r}+V_{2}
$$

где $V_{2}$ - произвольная функция, которая не может быть приведена к виду $P_{s}(r) e^{-b_{s} r}$.

Аналогично можно доказать следующую общую теорему.

ТЕОрема 4. Для того чтобъ функиия $f_{0}(-k, r)$ имела полюсъ порядка не въше $\left(n_{1}+1\right)$ в точках $i b_{1} / 2, i b_{1}, 3 i b_{1} / 2, \ldots$, не выше $\left(n_{2}+1\right)$ в точках $i b_{2} / 2, i b_{2}$, $3 i b_{2} / 2, \ldots$, не выне $\left(n_{m}+1\right)$ в точках $i b_{m} / 2, i b_{m}, 3 i b_{m} / 2, \ldots$, необходимо и достаточно, чтобы соответствующий потенциал содержал член $\sum_{m, n_{m}} P_{n_{m}}(r) e^{-b_{m} r}$.

Очевидно, для того чтобы иметь существенно особые точки, необходимо и достаточно, чтобы соответствующий потенциал содержал член $X(r) e^{-b r}$, где $X(r)$ - равномерно сходящийся бесконечный ряд вида $\sum_{n=0}^{\infty} \alpha_{n} r^{n}$, не равный $e^{\text {const. } r^{\alpha}}, 0<\alpha<\infty$.

Исследуя поведение интеграла $I$ вдоль оси $k=i b / 2$ в случае, когда $V(r)=$ $v(r) e^{-b r}$, где $v(r)$ - произвольная функция, не имеющая множителя $e^{\text {const } \cdot r^{\alpha}}, \alpha \geqslant 1$, легко заключить, что на этой оси могут появляться точки ветвления. Один из простейших случаев представляет собой потенциал $\left[e^{-b r} \sin (c r)\right] / r^{q}$. Для различных значений $q(q>0)$ этот потенциал может давать точки ветвления различных видов при $k=i b / 2 \pm c / 2$.

Если $I(r)=v(r) e^{-b r^{\alpha}}, \quad \alpha$ - целое число, $\alpha>1$, то $I$ и, следовательно, $f_{0}(k, r)$ будут аналитическими функциями на всей плоскости.

Таким образом, наличие множителя $e^{-b r}$ приводит к тому, что функция $f_{0}(-k, r)$ становится неаналитической в верхней полуплоскости.

\section{Вывод формулы (24)}

ПРИЛОЖЕНИЕ 2

Перепишем выражение $(6 \mathrm{a})$ для $f_{0-}(k, r)$ внутри окружности $\gamma_{\mathrm{c}}$, проведенной вокруг точки $k_{\gamma}=i b / 2$, в виде

$$
\begin{aligned}
f_{0-}(k, r) & \rightarrow \lim _{k \rightarrow k_{\gamma}}\left\{1+\rho\left[1+\frac{i \rho}{2 k} \ln \left(1+\frac{2 i k}{b}\right)\right]^{-1} \int_{b}^{\infty} \frac{e^{-b^{\prime} r}}{b^{\prime}\left(b^{\prime} \mp 2 i k\right)} d b^{\prime}\right\} e^{ \pm i k r}= \\
& =\lim _{\eta \rightarrow 0}\left\{1+\rho\left[1+\frac{i \rho}{2 k} \ln \left(1+\frac{2 i k}{b}\right)\right]^{-1} \int_{0}^{\infty} \frac{e^{-(\tilde{b}+b) r}}{(\tilde{b}+\eta)(\tilde{b}+b)} d \tilde{b}\right\} e^{-i k r},
\end{aligned}
$$


где мы ввели переменные $\tilde{b}=b^{\prime}-b$ и $\eta=2 i k+b$. Затем сделаем следующие простые преобразования правой части (П.7):

$$
\begin{aligned}
f_{0-}(k, r) \rightarrow & \lim _{\eta \rightarrow 0}\left\{1+\rho\left[1+\frac{i \rho}{2 k} \ln \left(1+\frac{2 i k}{b}\right)\right]^{-1} \int_{0}^{\infty} \frac{e^{-\tilde{b} r} e^{2 i k r} d \tilde{b}}{(\tilde{b}+\eta)(\tilde{b}-2 i k+\eta)}\right\} e^{-i k r}= \\
= & e^{-i k r}+\rho\left[1+\frac{i \rho}{2 k} \ln \left(1+\frac{2 i k}{b}\right)\right]^{-1} \int_{0}^{\infty} \frac{e^{-\tilde{b} r} d \tilde{b}}{\tilde{b}(\tilde{b}-2 i k)} e^{i k r}+ \\
& +\rho\left[1+\frac{i \rho}{2 k} \ln \left(1+\frac{2 i k}{b}\right)\right]^{-1} \lim _{\eta \rightarrow 0} \int_{0}^{b} \frac{e^{-\tilde{b} r} d \tilde{b}}{(\tilde{b}+\eta)(\tilde{b}-2 i k+\eta)} e^{i k r}= \\
= & e^{-i k r}+\frac{A_{-}}{A_{+}}\left[f_{0+}(k, r)-e^{i k r}\right]+ \\
& +\rho\left[1+\frac{i \rho}{2 k} \ln \left(1+\frac{2 i k}{b}\right)\right]^{-1} \lim _{\eta \rightarrow 0} \int_{0}^{b} \frac{e^{-\tilde{b} r} d \tilde{b}}{(\tilde{b}+\eta)(\tilde{b}-2 i k+\eta)} e^{i k r},
\end{aligned}
$$

где

$$
A_{\mp}=\left[1 \pm \frac{i \rho}{2 k} \ln \left(1 \pm \frac{2 i k}{b}\right)\right]^{-1}
$$

и мы воспользовались определением (6a) для $f_{0+}(k, r)$.

Теперь проанализируем последний интеграл в правой части формулы (П.8), используя формулы (3.352.1) и (8.214.1) из [12]:

$$
\begin{aligned}
J & =\lim _{\eta \rightarrow 0} \int_{0}^{b} \frac{e^{-\tilde{b} r} d \tilde{b}}{(\tilde{b}+\eta)(\tilde{b}-2 i k+\eta)}=\lim _{\eta \rightarrow 0} \frac{1}{b-\eta} \int_{0}^{b} e^{-\tilde{b} r}\left[\frac{1}{\tilde{b}+\eta}-\frac{1}{\tilde{b}+b}\right] d \tilde{b}= \\
& =\lim _{\eta \rightarrow 0}\left\{\frac{1}{b-\eta} e^{\eta r}[\operatorname{Ei}(-b r-\eta r)-\operatorname{Ei}(-\eta r)]-\frac{1}{b-\eta} e^{b r}[\operatorname{Ei}(-2 b r)-\operatorname{Ei}(-b r)]\right\}= \\
& =\lim _{\eta \rightarrow 0} \frac{1}{b}[-\ln (\eta r)+X(\eta, r)]
\end{aligned}
$$

где Еi - функция Эйри,

$$
X(\eta, r)=\sum_{k=1}^{\infty} \frac{(-b r+\eta r)^{k}}{k \cdot k !}-\sum_{k=1}^{\infty} \frac{(-\eta r)^{k}}{k \cdot k !}-e^{b r}\left[\sum_{k=1}^{\infty} \frac{(-2 b r)^{k}}{k \cdot k !}-\sum_{k=1}^{\infty} \frac{(-b r)^{k}}{k \cdot k !}\right]
$$

- аналитическая функция аргумента $\eta=b+2 i k$ в точке $\eta=0$ и внутри небольшой окружности $|\eta|<b$. Далее можно переписать (П.9) следующим очевидным образом:

$$
J=\frac{1}{b}\left\{\left[1+\frac{i \rho}{2 k} \ln \left(1+\frac{2 i k}{b}\right)\right] \frac{2 i k}{\rho}+Z(k, r)\right\}
$$

где $Z(k, r)=X(\eta, r)-\ln (b r)-2 i k / \rho$ - аналитическая функция переменной $k$ в точке $k=k_{\gamma}=i b / 2$ и внутри небольшой окружности $|k|<b / 2$. 
Используя выражение (П.9а), продолжим преобразования выражения (П.8):

$$
\begin{aligned}
f_{0-}(k, r) \underset{k \rightarrow k_{\gamma}}{\rightarrow} & e^{-i k r}+\frac{A_{-}}{A_{+}}\left[f_{0+}(k r)-e^{i k r}\right]+\rho\left[1+\frac{i \rho}{2 k} \ln \left(1+\frac{2 i k}{b}\right)\right]^{-1} \times \\
& \times \lim _{\eta \rightarrow 0} e^{i k r} \frac{1}{b}\left\{\left[1+\frac{i \rho}{2 k} \ln \left(1+\frac{2 i k}{b}\right)\right] \frac{2 i k}{\rho}+Z(k, r)\right\}= \\
= & W(k, r)+\left[1+\frac{i \rho}{2 k} \ln \left(1+\frac{2 i k}{b}\right)\right]^{-1} U(k, r),
\end{aligned}
$$

где

$$
\begin{gathered}
W(k, r)=e^{-i k r}+\frac{2 i k}{b} e^{i k r} \\
U(k, r)=\left[1-\frac{i \rho}{2 k} \ln \left(1-\frac{2 i k}{b}\right)\right]^{-1} f_{0+}(k, r)+e^{i k r}\left\{\frac{\rho}{b} Z(k, r)-\left[1-\frac{i \rho}{2 k} \ln \left(1-\frac{2 i k}{b}\right)\right]\right\}
\end{gathered}
$$

- аналитические функции аргумента $k$ в точке $k=k_{\gamma}=i b / 2$ и внутри небольшой окружности $|k|<b / 2$.

\section{Список литературы}

[1] Р. Ньютон, Теория рассеяния волн и частии, Мир, М., 1969.

[2] Х. М. Нуссенцвейг, Причинность и дисперсионные соотношения, Мир, М., 1976.

[3] В. С. Ольховский, ТMФ, 20:2 (1974), 211.

[4] М. В. Николаев, В. С. Ольховский, ТМФ, 31:2 (1977), 214.

[5] V. S. Olkhovsky, A. K. Zaichenko, Nuovo Cimento. A, 63 (1981), 155.

[6] В. С. Ольховский, Ю. В. Цехмистренко, Укр. физ. журнал, 6:2 (1961), 149. (поукраински).

[7] Е. К. Титчмарш, Теория функиий, Наука, М., 1980.

[8] T. Y. Tsang, T. A. Osborn, Nucl. Phys. A, 247 (1975), 43.

[9] В. С. Ольховский, ЭЧАЯ, 15 (1984), 289.

[10] R. Jost, Helv. Phys. Acta, 20 (1947), 256.

[11] S. Ma, Phys. Rev., 71 (1947), 195.

[12] И. С. Градштейн, И. М. Рыжик, Таблищъ интегралов, сумм, рядов и произведений, Физматгиз, М., 1963. 